\title{
Newly developed quantitative transactivation system shows difference in activation by Vitis CBF transcription factors on DRE/CRT elements
}

\author{
Annette Nassuth", Mahbuba Siddiqua, Huogen Xiao, Michelle A Moody and Chevonne E Carlow
}

\begin{abstract}
Background: Agroinfiltration-based transactivation systems can determine if a protein functions as a transcription factor, and via which promoter element. However, this activation is not always a yes or no proposition. Normalization for variation in plasmid delivery into plant cells, sample collection and protein extraction is desired to allow for a quantitative comparison between transcription factors or promoter elements.
\end{abstract}

Results: We developed new effector and reporter plasmids which carry additional reporter genes, as well as a procedure to assay all three reporter enzymes from a single extract. The applicability of these plasmids was demonstrated with the analysis of CBF transcription factors and their target promoter sequence, DRE/CRT. Changes in the core DRE/CRT sequence abolished activation by Vitis CBF1 or Vitis CBF4, whereas changes in the surrounding sequence lowered activation by Vitis CBF1 but much less so for Vitis CBF4. The system also detected a reduction in activation due to one amino acid change in Vitis CBF1.

Conclusions: The newly developed effector and reporter plasmids improve the ability to quantitatively compare the activation on two different promoter elements by the same transcription factor, or between two different transcription factors on the same promoter element. The quantitative difference in activation by VrCBF1 and VrCBF4 on various DRE/ CRT elements support the hypothesis that these transcription factors have unique roles in the cold acclimation process.

Keywords: CBF, DRE/CRT, Agroinfiltration normalization, Transactivation effector and reporter plasmids

\section{Background}

Transient transactivation systems have been developed to evaluate the activation of different promoters by transcription factors. They have been used successfully to analyze relative promoter strengths $[1,2]$, which are reportedly similar to those in transgenic systems [3]. Transient expression systems are preferred for the analysis of the sequence targeted by a transcription factor because genes that are directly activated by the transcription factor will produce transcripts within the time period between infiltration and harvest, whereas genes that are indirectly activated will take a longer time. Synthetic promoters containing (multiple copies of) defined regulatory elements are often used to avoid complications due to a combinatorial effect

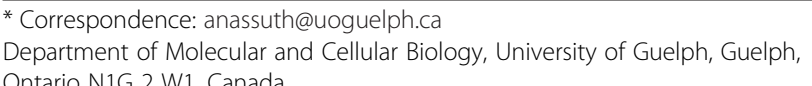

* Correspondence: anassuth@uoguelph.ca
Department of Molecular and Cellular Biology, University of Guelph, Guelph, Ontario N1G 2 W1, Canada
}

C Biomed Central

(c) 2014 Nassuth et al.; licensee BioMed Central Ltd. This is an Open Access article distributed under the terms of the Creative Commons Attribution License (http://creativecommons.org/licenses/by/4.0), which permits unrestricted use, distribution, and reproduction in any medium, provided the original work is properly credited. The Creative Commons Public Domain Dedication waiver (http://creativecommons.org/publicdomain/zero/1.0/) applies to the data made available in this article unless otherwise stated.

of various elements in a natural promoter, and have allowed the confirmation of cis-regulatory elements important for promoter activation by pathogens $[4,5]$.

DNA from promoter- and transcription factor-plasmids of interest have been introduced into plant cells by a wide variety of means but the most successful methods involve electroporation or PEG treatment for introduction into protoplasts [6-8], and particle bombardment [9,10] or agroinfiltration for introduction into plant tissues $[11,12]$. Studies on grape genes have employed particle bombardment of grapevine callus to investigate transactivation by transcription factors [13] and, with varying success, vacuum infiltration of grapevine leaves from in vitro grown plants to investigate gene function in fungal defense $[14,15]$ or agroinfiltration to study subcellular localization [16] or silencing constructs [17].

The CBF pathway in plants ultimately results in the expression of cold regulated (COR) genes which encode proteins that are thought to help the plant survive frost 
$[18,19]$. The name of the pathway derives from CBFs (CRT binding factors), the transcription factors initially discovered in Arabidopsis to be directly responsible for the activation of many COR genes at low temperatures by binding to CRT (defined as GCCGAC) elements in their promoters $[20,21]$. The same proteins were also discovered as DRE-binding transcription factors 1 (DREB1s), reported to bind to drought responsive elements (DRE; defined as TACCGACAT) [22,23]. As a result reference is often made to CBF/DREB1 factors (AtCBF1/DREB1B, AtCBF2/ DREB1A, AtCBF3/DREB1C) that bind to CRT/DRE elements with the sequence A/GCCGAC [18]. CBF/DREB1 proteins have now been reported for a wide variety of plants $[19,24]$ and these appear to also bind and activate via the CRT/DRE sequence. However, not all CBF proteins have the same affinity and specificity for a certain CRT sequence. For example, the Brassica napus BNCBF17 has a lower sequence binding specificity than BNCBF5 [25] whereas the barley HvCBF1 has a binding preference for an element, TTGCCGACAT, containing the GCCGAC (CRT) core sequence over a sequence with the ACCGAC (DRE) core [26]. The results with Chrysanthemum DREB1A- or $D R E B 1 B$-overexpressing Arabidopsis showed that these CBFs activate different, overlapping regulons, which is in agreement with preferences of these CBF-like proteins for different promoter elements [27]. Also analysis of the promoters from genes that were induced in AtCBF-overexpressing Arabidopsis revealed that variations in the sequence surrounding the CRT element might affect activation by various CBFs [28,29]. Together these results suggest that different CBF paralogs in a plant, and possibly orthologs from different species, have unique preferences for CRT-like sequences but more research is needed to investigate this further. Our lab successfully applied agroinfiltration of tobacco leaves to show that CRT promoter elements are required for regulation of gene expression by grape CBF transcription factors [30,31]. The results also suggested that CBF4 activates better than CBF1 however our analyses did not consider differences in infiltration and extraction that might occur between separate events.

The goal of the present study was to introduce an optimized dual luciferase reporter assay system that allows a better quantitative comparison of gene expression between different combinations of transcription factors (TFs) and promoter elements. The resulting system was used to analyze the activation by grape CBF1 and CBF4 on artificial promoters containing variations of the CRT sequence, and to compare the activation by $\mathrm{CBF} 1$ of the wild grape Vitis riparia ( $\mathrm{VrCBF} 1$ ) and a VrCBF1 with one amino acid mutated into the amino acid present in the CBF1 of the more freezing sensitive winegrape $V$. vinifera.

\section{Results}

Development of a quantitative dual luciferase transactivation system

Effector plasmids were prepared starting from pCAMBIA 1305.1 (Figure 1A). For the VrCBF4 effector plasmid (Figure 1B), this involved adding a 35S::VrCBF4-nos terminator cassette into the multiple cloning site (MCS). The VrCBF4 open reading frame (ORF) was replaced by the VrCBF1 ORF for the VrCBF1 effector plasmid. To prepare the reporter plasmid, the VrCBF4 effector plasmid was altered in several aspects. The GUSPlus ORF was replaced by the firefly luciferase (FiLUC) ORF, the VRCBF4 ORF was replaced by the renilla luciferase (RiLUC) ORF, and its $35 \mathrm{~S}$ promoter was replaced by a 4XCRTmin35S promoter (Figure 1C). As a result, the GUS activity can be taken as an indicator of the amount of effector plasmid, and the FiLUC activity as an indicator of the amount of reporter plasmid.

Various assay conditions were tested for compatibility with an analysis of the activities of the beta-glucuronidase, renilla luciferase and firefly luciferase reporter enzymes in a single extract. It was determined that extracts prepared in CCLR and diluted in PLB buffer (both from Promega) could be used for either glucuronidase or luciferase assays. Dilutions between 75 and $100 x$ gave values below the maximum value for the fluorescence reader and were in a linear range. This indicates that all reporter enzyme activity values could be determined from the same extract.

The GUS/protein and FiLUC/protein values were expected to be the same for each effector/reporter infiltration treatment if the amount of DNA taken up and expressed in leaves infiltrated with VrCBF4 effector and reporter plasmid-containing agrobacteria (reporters containing the M1, M2, M4 and M5 variants of the DRE/CRT element, for details on these variants see a later section), was similar. The results show that both GUS/protein and FiLUC/protein values vary (Figure 2A), indicating that the amount of DNA transferred and expressed in the plant cells after the different infiltrations varied for both effector (GUS) and reporter (FiLUC) plasmid. Also the FiLUC/GUS values vary (Figure 2B), indicating that the ratio between effector DNA and reporter DNA uptake and expression varies. This means that the amounts of $\mathrm{CBF}$ protein and reporter gene in the cells might vary independently from each other, and this can cause variations in activation between separate infiltrations. The RiLUC/FiLUC ratio can therefore not be taken as a true measure of transactivation (Figure 2C). Instead it is better to normalize for the amount and expression of effector plasmid (represented by GUS) as well and therefore the RiLUC/FiLUC/GUS was taken as a measure of transactivation (Figure 2D). 

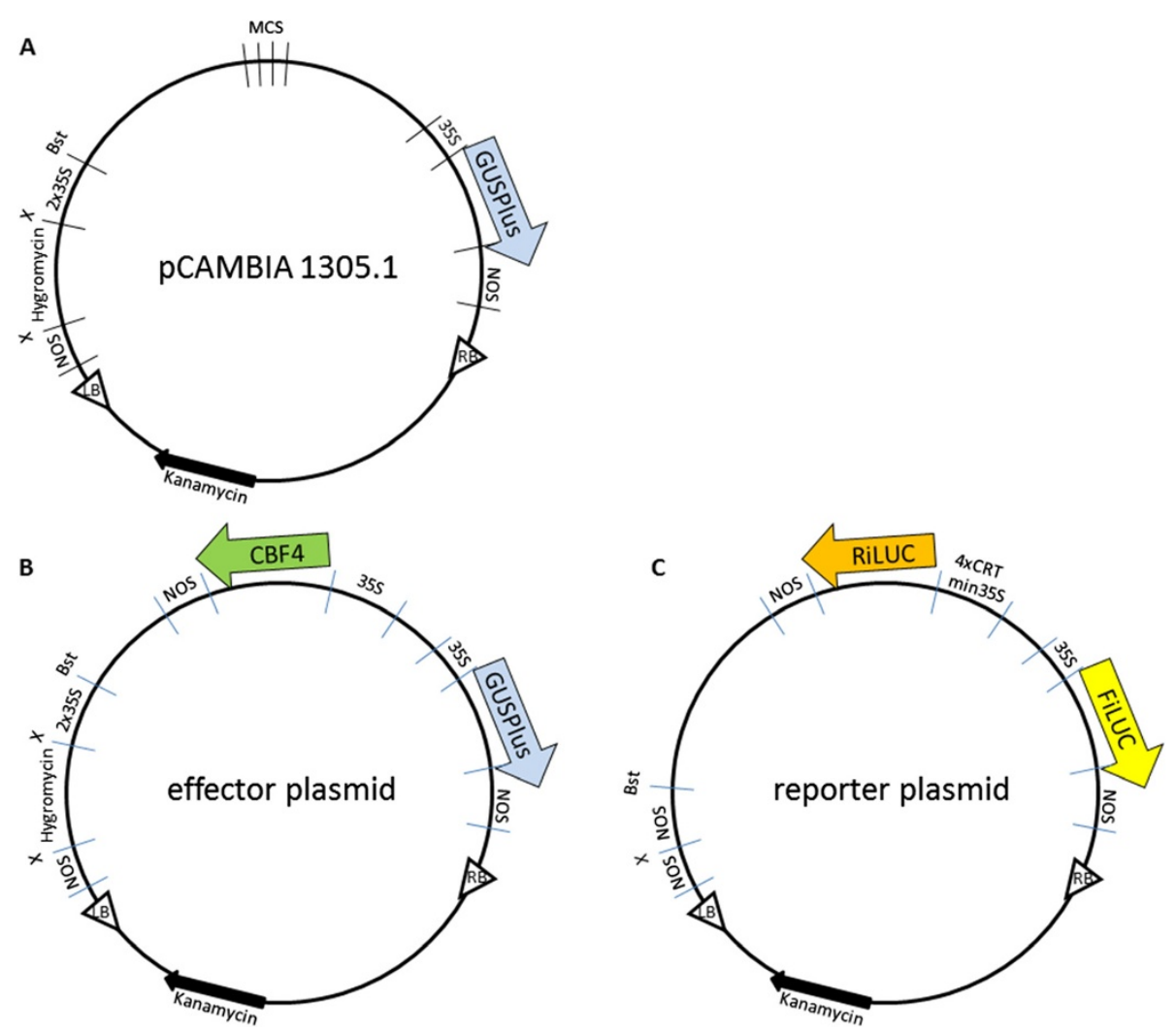

Figure 1 Schematic representation of $[A]$ pCAMBIA1305.1, [B] GUS effector and [C] dual luciferase reporter plasmids. MCS $=$ multiple cloning site. See Materials and Methods text for further details.

\section{VrCBF1 and VrCBF4 require the conserved DRE/CRT core sequence to transactivate}

To determine whether the transactivation by VrCBF1 and $\mathrm{VrCBF} 4$ has strict requirements regarding the TACCGACAT sequence present in the RiLUC reporter gene promoter, various mutations were made in this sequence and tested for transactivation. Examination of the results with the regular CRT sequence $(\mathrm{GCCGAC}=\mathrm{M} 2)$ showed that both $\mathrm{VrCBF} 1$ and $\mathrm{VrCBF} 4$ gave higher RiLUC/FiLUC/GUS values, respectively about 6 and 14 times higher, than the values obtained in the absence of a CBF (Figure 3). Mutations in the CCGAC core DRE/CRT sequence significantly reduced the activation by $\operatorname{VrCBF} 1$ and $\mathrm{VrCBF} 4$ to values that were not statistically different from those obtained without CBF.

The DRE sequence is ACCGAC, whereas the CRT sequence has a $\mathrm{G}$ instead of an $\mathrm{A}$ to give GCCGAC [20-23]. The question that was posed is whether the different Vitis CBFs have different affinities for these 2 sequences, and whether another change of the initial nucleotide has an effect. Figure 4 shows that $\mathrm{VrCBF} 1$ and VrCBF4 activated reporters with either TACCGACAT (M1) or TGCCGACAT (M2) elements to similar levels, but when the first nucleotide (A or $\mathrm{G}$ ) is mutated to C (M10:CCCGAC) or T (M11:TCCGAC) the RiLUC/ FiLUC/GUS values drop to control levels. This result, and also the results from Figures 3 and 5 support our previous suggestion that CBF4 activates better than CBF1 [31]. Five independent replicates of this experiment showed a higher activation by VrCBF1 on CRT (M2) over that on DRE (M1), and this difference was significant in three experiments (see also Figure 5).

\section{Nucleotides flanking DRE sequence also affect activation levels}

Nucleotides around the DRE sequence were changed to determine if such changes affect transactivation levels. These sequence variants were made to reflect CRT/DRE elements in Arabidopsis genes that had been reported targets of DREBs. These included elements found in genes as reported by Seki and colleagues [28], namely RD29A/COR78, (M1: TACCGACAT), RD17/COR47 (M3: GACCGACAT) and RD17/COR47 (M4: TACC GACTT). Two other chosen variants were based on the frequency logo determined for cold responsive genes, as reported by Wang and colleagues [32] (M5: GACCGACAA) or drought responsive genes (M6: GACCGACTC). In the 

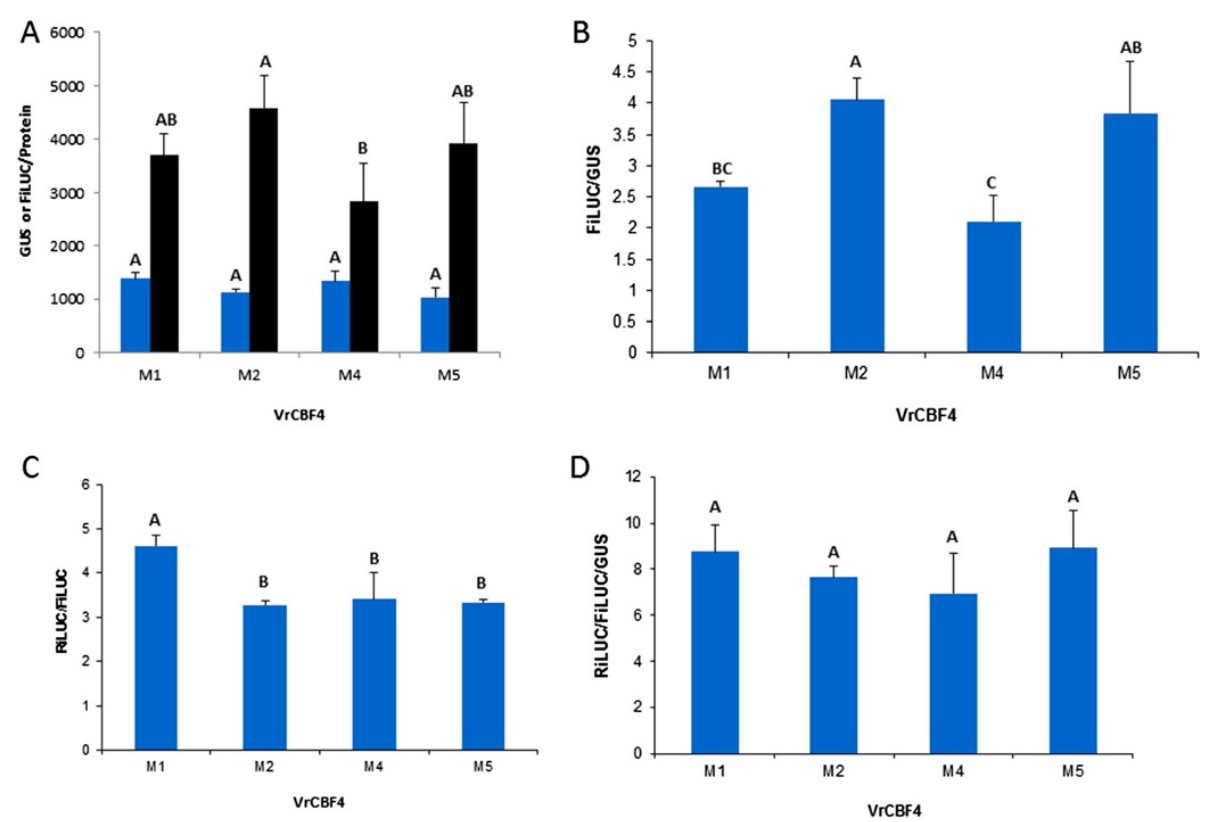

Figure 2 Analysis of reporter gene expression after agroinfiltration of tobacco leaves with $\operatorname{VrCBF} 4$ effector in combination with four different 4XCRTmin35S reporter constructs. [A] GUS/protein (blue bars) and FiLUC/protein (black bars), [B] FiLUC/GUS, [C] RiLUC/FiLUC, [D] RiLUC/FiLUC/GUS. Shown are the averages of three technical replicates and their standard deviation. Different letters indicate statistically significant differences (ANOVA $p<0.05$ ).

absence of CBF effector, M1 to M6 gave variable activation values which were higher than background activation values with M7, the negative control CRT variant (Figure 5). Even higher activation values were obtained with VrCBF1 or VrCBF4 on all CRT variants except for the negative control M7. Both VrCBF1 and VrCBF4 activation on sequence variant $\mathrm{M} 2$ was among the highest whereas activation on sequence variant M4 was the lowest in all experiments and this was significant in two out of three independent experiments (Figure 5). However, activation by VrCBF1 was more affected by nucleotide changes around the DRE/CRT sequence than activation by $\mathrm{VrCBF} 4$ which resulted, generally speaking, in higher induction of transcription by $\mathrm{VrCBF} 4$ compared to VrCBF1 irrespective of the DRE/CRT variant present in the reporter plasmid.

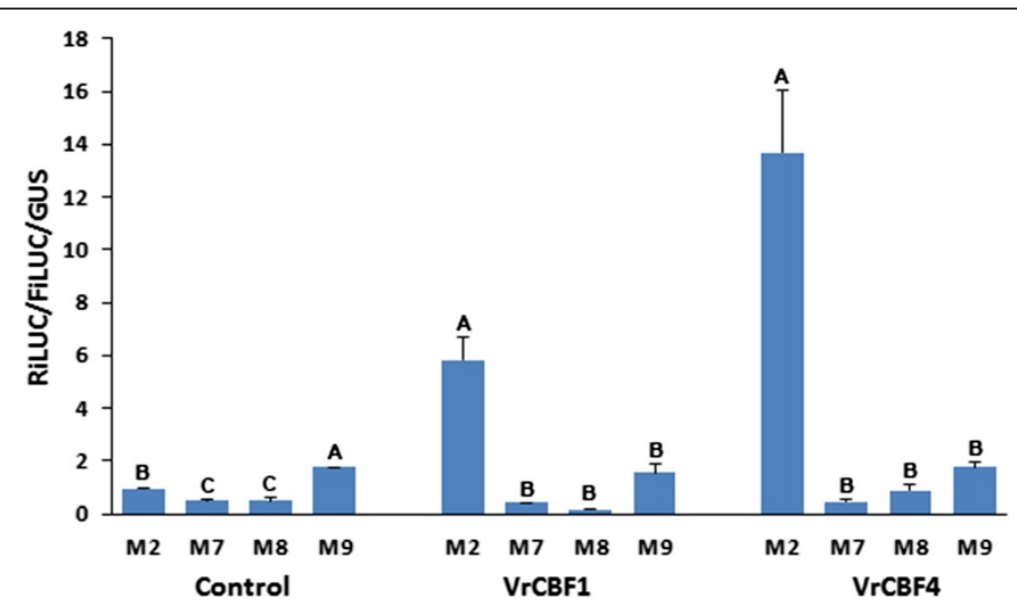

Figure 3 Activation by VrCBF1 or VrCBF4 on CRT (M2) and mutated core CRT sequence. M2: TGCCGACAT, M7: TGAAGACAT, M8: TGCCGCCAT, M9: TGCCGAAAT. Error bars represent the standard deviation. Infiltrations without CBF effector were included as control. Statistical analysis was performed on the set of no CBF, VrCBF1 and VrCBF4 data separately and significantly different activation values (ANOVA p < 0.05) are indicated by different letters. Similar results were obtained for two other independent experiments. 


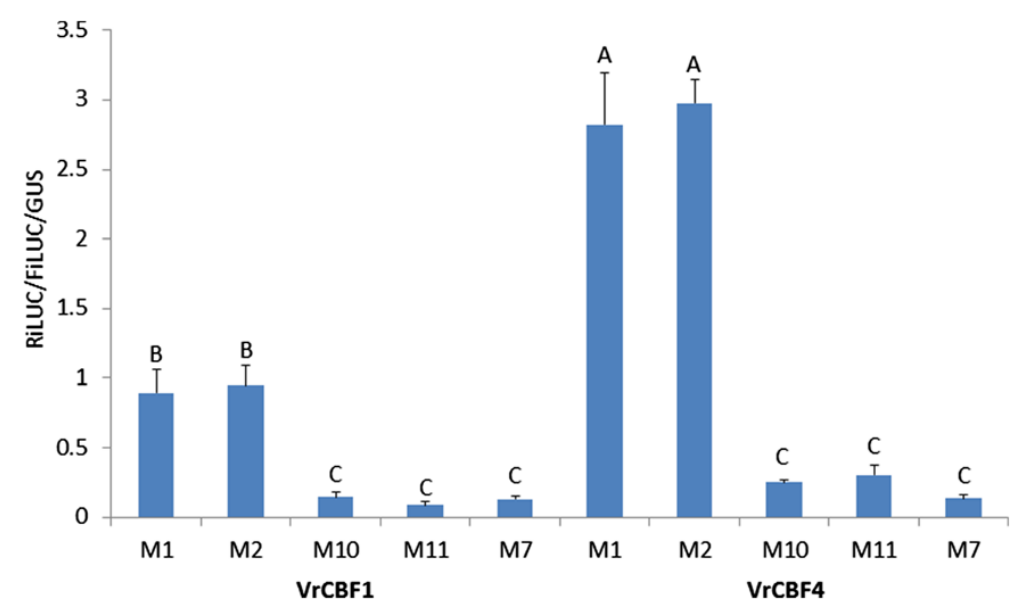

Figure 4 Activation by VrCBF1 or VrCBF4 on a reporter with various initial nucleotides in the DRE/CRT sequence. M1 = DRE: TACCGACAT, M2 = CRT: TGCCGACAT, M10: TCCCGACAT, M11: TTCCGACAT (core CRT/DE sequence in italics), and the negative control CRT variant M7: TGAAGACAT Statistical analysis was performed on the set of no CBF, VrCBF1 and VrCBF4 data separately and significantly different activation values (ANOVA $p<0.05$ ) are indicated by different letters. Error bars represent the standard deviation. Similar results were obtained in three other independent experiments.

\section{Change in CBF amino acid sequence affects activation levels}

Based on the higher transactivation by CBF1 from $V$. riparia compared to activation by CBF1 from $V$. vinifera in our previous transactivation system [30], we predicted that a change of the glutamic acid (E) in the AP2 DNA binding domain of $\mathrm{VrCBF} 1$ at position 85 to a lysine (K), as is present in $\mathrm{VvCBF} 1$, would decrease the transactivation. Therefore, transactivation by $\mathrm{VrCBF} 1$ was compared to that by the mutant VrCBF1-E85K in the newly developed dual luciferase transactivation system, on the two DRE/CRT variants that had given the highest values (M2 and M5, see Figure 5). An empty control effector plasmid was included to confirm that the activations observed with either CBF1 or CBF4 are higher than those observed in the presence of endogenous tobacco transcription factor only (control). The results showed that the mutant VrCBF1. E85K indeed had a lower activation of the reporter with either the M2 or M5 variants (4.0x or $1.1 \mathrm{x})$, as compared to the wild type $\operatorname{VrCBF} 1$ (5.0x or 2.2x) (Figure 6). This difference in activation was observed in three independent experiments and was statistically significant in two of these.

\section{Discussion}

New effector and reporter plasmids were developed for transactivation analyses in plant tissues. The advantages of this new transactivation system are: (1) Two reporter genes, GUSPlus and FiLUC, were included in the vector

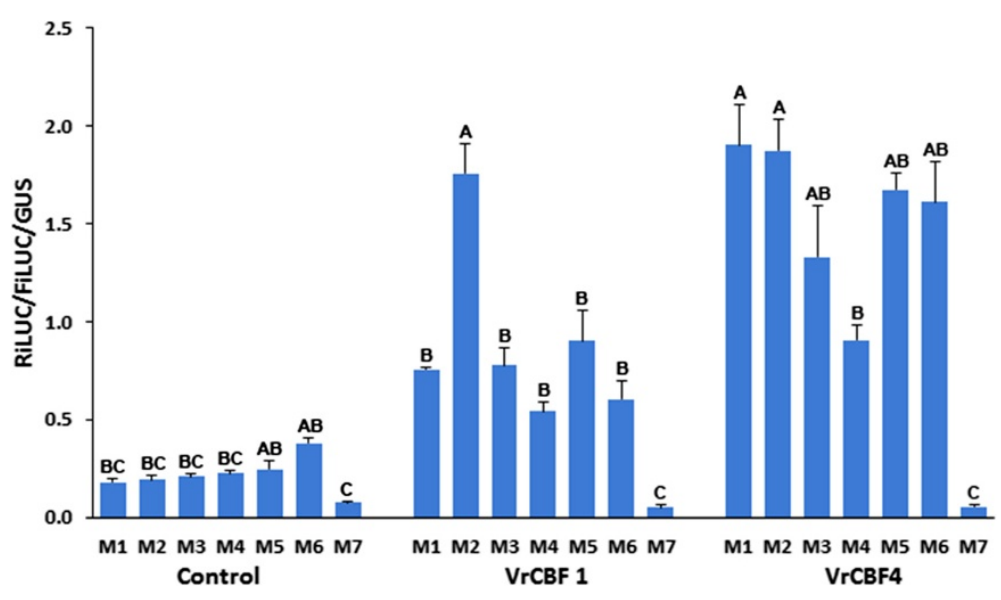

Figure 5 Activation by $\operatorname{VrCBF} 1$ and $\mathrm{VrCBF} 4$ on promoters with nucleotide variations around the DRE sequence (in italics). $M 1=\mathrm{DRE}$ : TACCGACAT, M2 = CRT: TGCCGACAT, M3: GACCGACAT, M4: TACCGACTT, M5: GACCGACAA, M6: GACCGACTC. The M7 CRT variant (TGAAGACAT) was included as a negative reporter control, whereas mixtures without a CBF were included as a negative effector control. Error bars represent the standard deviation. Statistical analysis was performed on the set of no CBF, VrCBF1 and VrCBF4 data separately and significantly different activation values (ANOVA $p<0.05$ ) are indicated by different letters. Similar results were obtained in two other independent experiments. 


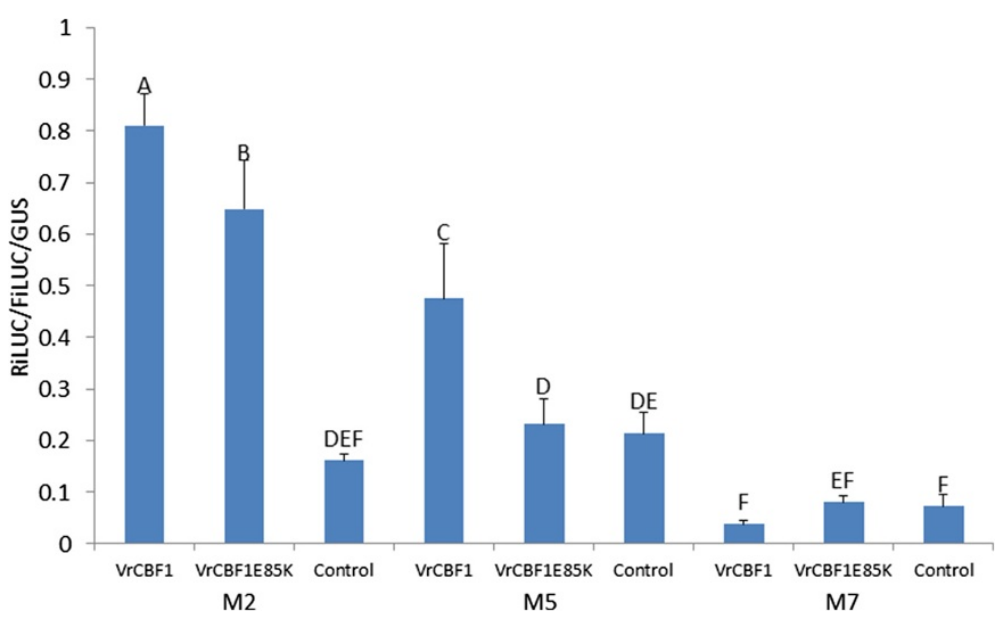

Figure 6 Comparison of activation by VrCBF1and VrCBF1E85K on the M2 and M5 CRT/DRE variants. M2 = CRT: TGCCGACAT, M5: GACCGACAA. The CRT variant M7 (TGAAGACAT) was included as a negative reporter control, whereas an empty effector was included as a negative effector control. Error bars represent the standard deviation. Statistical analysis was performed on all data together and significantly different activation values (ANOVA $p<0.05$ ) are indicated by different letters. Similar results were obtained in two other independent experiments.

plasmids to be able to normalize for the amount of these proteins and be used as an indicator for the amount of respectively effector and reporter DNA transferred and expressed in the leaf cells. Other researchers examining either plant or animal systems have included a constitutively expressed reporter gene (35S::LUC cassette) on a separate plasmid $[33,13,34]$ to normalize for differences in infiltration between samples. This was under the assumption that both plasmids are delivered in similar quantities into the cells. Our results show that this assumption is not true (Figure 2). (2) The chosen reporter genes have an intron, which cannot be spliced out by bacteria [35], therefore no enzyme is translated from erroneous transcripts that might be produced in the numerous Agrobacteria present in the infiltrated leaf tissue [36]. Commonly used reporter genes for plant tissues are $\beta$-glucuronidase (GUS) $[37,38]$, green fluorescent protein (GFP) $[13,39,40]$, firefly (Photinus pyralis) luciferase (FLUC) [41], and sea pansy (Renilla reniformis) luciferase (RLUC) [42]. The understanding that the inclusion of an intron is important led to the development of intron-containing reporters such as GUSi [34], GUSINT [36], GUSPlus (CAMBIA, Canberra, Australia and [30]), FiLUC [43] and RiLUC [44]. (3) Beta glucuronidase (GUSPlus), renilla luciferase (RiLUC) and firefly luciferase (FiLUC) were chosen as reporters as they all can be measured by a similar procedure. We avoided green fluorescent protein (GFP) as a reporter, since this can diffuse out of the cell [9]. Previously, Renilla luciferase has been used as the normalizer [2], but studies have shown that this luciferase has a 100 fold higher signal when compared to firefly luciferase [45] which gives it a wider range. Therefore, RiLUC was chosen to quantify differences in activation for our system. (4) The quantification procedure uses the same extract for the analysis of all reporter activities. This means that the activation value, RiLUC/FiLUC/GUS, is normalized for variation that may occur through infiltration, DNA uptake or protein extraction. The fact that new substrate solution for all enzymes has to be prepared fresh for every experiment means that some variation will exist between experiments and this can affect activation values. We therefore suggest that only effector and reporter combinations that have been analyzed in the same experiment be compared to each other.

The reporter construct was designed to contain 4 DRE/CRT sequence repeats combined with a minimal $35 \mathrm{~S}$ CaMV promoter. The $46 \mathrm{nt}$ minimal $35 \mathrm{~S}$ promoter is one of the best characterized plant core regulatory promoter domains [46,47] with a reportedly very low basal transcription level in the absence of additional upstream regulatory elements [48], and has already been used successfully in our previous experiments [30,31]. Although activation by transcription factors of promoters containing only 1 binding domain was shown to be detectable [49], adding additional (4) repeats of a DRE/CRT element was considered appropriate since it would give a stronger activation and therefore higher reporter enzyme activity which would make differences in activation efficiency easier to detect [4]. The low level of activation on mutant DRE/CRT reporter constructs (Figures 3 and 4) showed that there is no significant contribution from any other potential enhancer elements on the reporter vector (for example, in the $35 \mathrm{~S}$ promoter) to the RiLUC reporter activities caused by the Vitis CBFs. The low RiLUC/FiLUC/ GUS values on various DRE/CRT variants suggests that 
there is some background activation by tobacco transcription factors especially when compared to the values on the M7 mutant CRT/DRE sequence, when no grape CBF-producing plasmid was included (see especially M6 and M9, Figures 3 and 5).

An advantage of a transient transactivation system is that one can detect an increase in transcripts from genes that are directly activated by the transcription factor under study, even if this activation is temporary and therefore not detectable in transgenic plants. Another advantage is that transcripts of indirectly activated genes, which can be detected in transgenic plants, are likely absent in the transient system. The transient expression system can therefore assist to interpret results from transgenic plants. For example, we previously reported that compared to wild type Arabidopsis, VrCBF4- but not VrCBF1-overexpressing plants have increased AtRGL3 expression [50]. One might speculate that this is due to a preference by $\mathrm{VrCBF} 4$ and not $\mathrm{VrCBF} 1$ for the DRE/CRT-like sequence CCGCC in the AtRGL3 promoter. However, the presented transient expression results showed that the M8 reporter construct (containing CCGCC) is not activated much by either VrCBF1 or VrCBF4. Similarly, because VrCBF1- but not VrCBF4-overexpressing plants had an increased RD29A (COR78) expression, it could be argued that this was due to a preference by $\mathrm{VrCBF} 1$ and not $\mathrm{VrCBF} 4$ for the CCGAC sequence present twice in the promoter of this gene [50]. However, the transient expression system results shows that all reporter constructs containing CCGAC (M1 to M6) are activated better by $\mathrm{VrCBF} 4$ than by $\mathrm{VrCBF} 1$ (Figures 4 and 5). This suggests that the induction of AtRGL3 or RD29A in the transgenic Arabidopsis is due to an indirect effect, although it is also possible that the CBFs activate these genes via a DRE/CRT element outside of the "promoter" region that was examined for sequence elements, about $1 \mathrm{~kb}$ upstream of the ATG start codon [50].

All presented agroinfiltrations were performed in tobacco leaves, by a relatively easy procedure. We were not successful in infiltrating leaves from grapes grown under growth chamber conditions, despite trying various methods including vacuum infiltration. Indeed, also other researchers reported their failure to do so and were only successful if in plants were grown in vitro $[14,15]$. This has not been pursued further at this time since in vitro culture is labour-intensive and transactivation in grape leaves would only be necessary if one wanted to analyze the transactivation of endogenous grape genes.

Changes in the core DRE/CRT sequence greatly reduced the transactivation values (Figures 3 and 4), confirming that the complete core DRE/CRT sequence is required for binding by Vitis CBF1 or 4. This is in line with the report that Arabidopsis DREB1A (AtCBF3) and DREB2A bind weakly or not at all when the core CRT sequence is altered
[51]. Binding by the Arabidopsis DREB proteins was not affected by changes in the surrounding sequence [51] however our transactivation results show that this is different for the Vitis CBFs, especially for Vitis CBF1 (Figure 5). Also of note, is that CRT variant M8 contains the core sequence of the GCC box (GCCGCC) which is known to interact with ERF transcription factors of the ethylene signalling pathway [52] but not with Arabidopsis DREB proteins [51] and, as shown here, also not with Vitis CBF1 and CBF4 (Figure 3).

Inclusion of the VrCBF4 effector plasmid generally resulted in higher activation of the DRE/CRT variants than inclusion of the VrCBF1 effector plasmid (Figures 4 and 5). This supports our suggestion that VrCBF4 is a better activator than VrCBF1 on CRT variant M1 based on previous experiments using a different transactivation system [31]. In principle, there are several possible explanations for this phenomenon besides an inherent better activation capability for the $\mathrm{VrCBF} 4$ protein. It is possible that different amounts of protein are produced for each because, even though we used the same 5'UTR and 3'UTR sequences for each construct, the coding sequence can also affect translation efficiency [53]. Other possible explanations include a difference in the half-life of the RNA or protein. Translation efficiency and RNA or protein stability likely differ between different tissues and conditions (e.g. ambient vs cold treatment), and the situation found here in tobacco leaves might therefore not reflect the conditions that exist when $\mathrm{VrCBF} 1$ and $\mathrm{VrCBF} 4$ are expressed in grape tissues. Quantification of CBF protein levels would be possible by Western blot analysis with antibodies specific for each CBF or to a tag added to each CBF. However, the results of the experiment shown in Figure 5 suggest that possible differences in protein quantity are not the main reason for the observed differences in activation by VrCBF1 and VrCBF4. In this experiment the same experimental parameters (bacterial cultures, tobacco plants, length of incubation etc.) were used for all reporters but not all reporters show a lower activation with VrCBF1 than with VrCBF4. A more likely explanation for these results is that VrCBF1 has a preference for the M2 sequence whereas $\mathrm{VrCBF} 4$ is more promiscuous. The higher activation by $\mathrm{VrCBF} 1$ vs. VrCBF1E85K supports the hypothesis that this amino acid difference contributes to the difference in freezing tolerance between $V$. riparia and $V$. vinifera. The ability to detect this difference shows the sensitivity of the transactivation system to detect changes in activation due to single amino acid sequence differences.

\section{Conclusions}

Here we describe the development of a novel set of effector and reporter plasmids for transient expression studies using agroinfiltration. The use of intron-containing reporter 
genes allow for normalization of transactivation values for variation in plasmid entry into the plant cells, sample collection and extract preparation. The ability to distinguish between activation by plasmids with minor sequence variations in DRE/CRT promoter elements or a CBF transcription factor suggests that this system could be valuable in examining a variety of transcription factors and their putative target promoter sequences. The results with VrCBF1 and VrCBF4 activation on DRE/CRT variants suggests that these two transcription factors likely activate different overlapping sets of genes, and therefore have unique roles in cold acclimation.

\section{Materials and methods \\ Preparation of effector and reporter plasmids}

The pCAMBIA 1305.1 binary vector containing a multiple cloning site (MCS) and a 35S::GUSPlus reporter gene (http://www.cambia.org/daisy/cambia/585.html), a gene with a catalase intron, was taken as the starting point for the creation of effector constructs first (Figure 1A). This is a multicopy plasmid, in contrast to the previously used pBI121 [30,31], and thus easier to use. The original pCAMBIA plasmid, which does not encode any CBF, was used as a negative control effector. A HindIII/EcoRI cassette containing a $35 \mathrm{~S}$ promoter, $\mathrm{VrCBF} 4$ coding region with 5' ribosome binding site (rbs), and Nos terminator sequence, obtained from a previously prepared pBI121-based effector [31], was inserted into the MCS of pCAMBIA, yielding the 35S::VrCBF4 pCAMBIA effector (Figure 1B). Preparation of 35S::VrCBF1 pCAMBIA effector plasmid involved simply replacing the BamHI/SacI fragment containing the $5^{\prime}$ ribosome binding site and VrCBF4 coding sequence [31] with a similar fragment containing VrCBF1 coding sequence [30].

The reporter construct was prepared from these pCAMBIA effector constructs in several steps. First the GUSPlus reporter sequence was replaced with a FiLUC (Firefly luciferase coding sequence including the PIV intron from GUS $^{\mathrm{INT}}$ ) reporter sequence. To this end, NcoI/PmlI digested pCAMBIA effector, i.e. without the GUSPlus sequence, was ligated to an $\mathrm{NcoI} / \mathrm{PmlI}$ fragment containing the FiLUC sequence which had been amplified from pLUC07 [42] using primers that introduce these restriction sites (FiLUC-H-2 + NcoI: 5'AGGTAAGCCATGGAAGA CGCCAA 3' and FiLUC-C1842 + PmII: 5'TACACGTGT TACAATTTGGACTTTCCGC 3'). Second, the VrCBF4 coding region was replaced with the RiLUC reporter (Renilla reniformis luciferase coding sequence including a modified intron from the castor bean catalase gene). This was accomplished by ligating a BamHI/SacI RiLUC fragment amplified from RiLUC plasmid [44] using primers that introduce these restriction sites (RiLUCH1 + BamHI: 5'ATGGATCCAAGGAGATATAACAAT GACTTCGAAAGTTTATGATCC 3' and RiLUC-C936 +
SacI: 5'CGTTGACGAGCTCTTATTGTTCATTTTTGA GAACTCG 3') to BamHI and SacI digested, FiLUC containing pCAMBIA, similar to the CBF fragments earlier. Third, vectors with the RiLUC reporter driven by a $4 x C R T$ min35S promoter, consisting of $4 \mathrm{x}$ TACCGACAT [30] plus 46 nucleotides of the $35 \mathrm{~S}$ promoter $[46,47]$, were constructed. To this end the HindIII-BamHI 35S promoter-containing fragment was replaced with a HindIII-BamHI fragment containing 4xCRTmin35S promoter (obtained from plasmids described in [30]). Finally, the 2x35S::hygromycin fragment was deleted by digestion with $\mathrm{XhoI}$ and BstXI and replaced by a nos promoter fragment amplified from pBI121 using primers that introduce these restriction sites (NosproH1+ BstXI: 5' ACCACCATGTTGGGATCATGAGCGGAGAA TTAAG 3' and NosproC307 + Xhol: 5' GCAGGCTCGA GAGATCCGGTGCAGATTATTT 3'), and the completed reporter plasmid was ready for use (Figure $1 \mathrm{C}$ ). Effector and reporter plasmids were introduced into $A$. tumefaciens strain EHA105 according to the freeze-thaw method described by Höfgen and Willmitzer [54].

Reporter plasmids with altered CRT sequences were prepared by a quick change protocol essentially according to the procedure described by Stratagene on a smaller "35S cloning" plasmid [30] and subsequent subcloning of the new promoter fragment into the reporter plasmid. All primers that were used to prepare the various reporter plasmids are listed in Additional file 1: Table S1.

\section{Agroinfiltration of tobacco leaves}

Agroinfiltration was performed based on the protocols developed by Bendahmane and colleagues [55] and Vaucheret [56], essentially as described previously [30,31]. Nicotiana benthamiana plants were grown at $22^{\circ} \mathrm{C}$ for 16 hours of light and $20^{\circ} \mathrm{C}$ for 8 hours of dark until they reached a six leaf stage (approximately 4 weeks). Equal volumes of $A$. tumefaciens with reporter construct and of A. tumefaciens with effector construct were mixed to produce a final OD600 of 0.5 for each construct and the youngest two fully expanded leaves (leaves 3 and 4) of three different plants were infiltrated from the abaxial (lower) side with this mixture using a syringe. After 40-hour co-cultivation, one disc was taken from each infiltrated leaf, for a total of 6 discs per condition, frozen in liquid nitrogen and stored at $-80^{\circ} \mathrm{C}$. This was repeated 3-4 times to obtain 3-4 biological replicates for each mixture. Each experiment was repeated at least 2 times.

\section{Preparation of extracts}

Several protocols were tested for the extraction of leaf tissue to identify a procedure that is compatible with both glucuronidase and luciferase activity measurements, so that all reporter enzymes could be analyzed for the same 
extract. The harvested, frozen leaf discs were ground to powder with liquid $\mathrm{N}_{2}$ and then $9 \mu \mathrm{l}$ extraction buffer was added per $\mathrm{mg}$ tissue $(300 \mu \mathrm{l}$ buffer for six $\sim 6 \mathrm{~mm}$ leaf discs). As extraction buffer we tried either GUS extraction buffer $(25 \mathrm{mM}$ potassium phosphate $\mathrm{pH} 7.8,1 \mathrm{mM}$ EDTA, $7 \mathrm{mM}$ 2-mercaptoethanol, 1\% Triton X-100, and $10 \%$ glycerol) or $1 \mathrm{X}$ Cell Culture Lysis Reagent (CCLR; Luciferase assay systems, Promega). The glucuronidase and luciferase activities determined for the extracts were more consistent and higher when CCLR had been used for the preparation and PLB (Promega) for the dilution of the extracts, and therefore CCLR and PLB were used for all further experiments. Extracts were incubated on ice for 1 hour and cell debris was pelleted by centrifugation for $10 \mathrm{~min}$ at $13000 \times \mathrm{g}$ (SpeedFuge ${ }^{\circ}$ SFR13K, Savant). The supernatant was then diluted $75 \mathrm{x}$ with PLB and used for protein, glucuronidase and luciferase assays.

\section{Analysis of protein quantities}

Protein quantities were determined using the Bio-Rad dye-binding assay essentially according to the procedure described by the manufacturer (Bio-Rad), based on the method of Bradford [57], with BSA as standard.

\section{Analysis of GUS, RiLUC and FiLUC activities}

GUS activity was determined after incubating a mixture of $10 \mu \mathrm{l}$ of the $75 \mathrm{x}$ diluted sample and $90 \mu \mathrm{l}$ of Assay buffer $(50 \mathrm{mM}$ pH 7 Sodium phosphate buffer, $10 \mathrm{mM}$ EDTA, 0.1 Triton X-100, $0.1 \%$ N-Lauroylsarcosine Sodium Salt, 10 mM 2-mercaptoethanol, 40 mM 4-MUG) for 30 minutes at $37^{\circ} \mathrm{C}$. Each reaction was stopped by adding $900 \mu \mathrm{l}$ of $0.2 \mathrm{M} \mathrm{Na}_{2} \mathrm{CO}_{3}$ and fluorescence caused by the conversion of 4-MUG to MUG was measured in a polystyrene flatbottom 96-well plate (Sarstedt) using a POLARStar Omega (BMG Labtech) microplate reader with the excitation filter set at $360 \mathrm{~nm}$ and the emission filter at 460-10, and orbital shaking at $300 \mathrm{rpm}$. Each extract was analyzed in triplicate and the average measurement was taken as the value for one replicate.

The dual luciferase protocol based on the Dual Luciferase reporter assay system from Promega, as reported by Cazzonelli and colleagues [43], was used essentially unchanged to quantify the amount of RiLUC and FiLUC expression. $75 \mathrm{x}$ diluted sample $(10 \mu \mathrm{l})$ was added to Luciferase reagent II (50 $\mu \mathrm{l}$, LARII) in a polystyrene flatbottom 96-well plate (Sarstedt) and mixed by pipetting. FiLUC fluorescence, indicative of FiLUC gene expression, was measured immediately in a POLARStar Omega microplate reader with the setting on Luminescence (end point). To measure RiLUC fluorescence, indicative of RiLUC gene expression, $50 \mu \mathrm{l}$ of Stop and Glo reagent (20 $\mu \mathrm{l}$ substrate into $1 \mathrm{ml} \mathrm{S \& G}$ buffer and mixed well, freshly prepared according to the instructions from Promega) was added to each sample, mixed by pipetting and returned to the luminometer for a second measurement. Both measurements were set on $0.2 \mathrm{sec}$ delay with 10 flashes per well. Activities for all replicates of each extract were measured consecutively before a second set of replicates was prepared and measured.

Transactivation was expressed as RiLUC/FiLUC/GUS $x$ 100000. The resulting data were analyzed by a one-way analysis of variance (ANOVA). Statistical differences amongst the means for each reporter/effector combination within an experiment were determined by the Tukey-Kramer HSD tests $(\mathrm{P}<0.05)$ using JMP (version 11.1.1; SAS Institute) statistical software.

\section{Additional file}

Additional file 1: Table S1. List of primers used to prepare effector and reporter constructs.

\section{Competing interests}

The authors declare that they have no competing interests.

\section{Authors' contributions}

MAM prepared and tested the original effector and reporter constructs, and participated in the development of the assays, MS participated in the development of the constructs and assays and performed the statistical analyses, HX participated in the preparation of some constructs and subsequent assays, CC participated in the assays and statistical analyses, AN conceived the study, participated in its design and coordination, and wrote the initial manuscript. All authors read and approved the final manuscript.

\section{Acknowledgements}

The authors thank Jeff Velten and Luc Mankin for a vector with the RiLUC sequence, and FiLUC sequence respectively. Zamir Jetha provided technical support developing extraction and dilution protocols optimal for reporter enzyme assays. M. Atikur Rahman assisted with the construction of the M10 and M11 reporter constructs. This work was funded by grants from ORF and NSERC to AN. CC was partially supported by an Ontario Graduate Fellowship.

Received: 7 July 2014 Accepted: 30 September 2014

Published: 3 October 2014

\section{References}

1. Chiera JM, Bouchard RA, Dorsey SL, Park E, Buenrostro-Nava MT, Ling PP, Finer JJ: Isolation of two highly active soybean (Glycine max (L.) Merr.) promoters and their characterization using a new automated image collection and analysis system. Plant Cell Rep 2007, 26:1501-1509.

2. Hellens RP, Allan AC, Friel EN, Bolitho K, Grafton $K$, Templeton M, Karunairetnam S, Gleave AP, Laing WA: Transient expression vectors for functional genomics, quantification of promoter activity and RNA silencing in plants. Plant Methods 2005, 1:13.

3. Hernandez-Garcia CM, Martinelli AP, Bouchard RA, Finer JJ: A soybean (Glycine max) polyubiquitin promoter gives strong constitutive expression in transgenic soybean. Plant Cell Rep 2009, 28:837-849.

4. Rushton PJ, Reinstaedler A, Lipka V, Lippok B, Somssich IE: Synthetic plant promoters containing defined regulatory elements provide novel insights into pathogen- and wound-induced signalling. Plant Cell 2002, 14:749-762.

5. Liu W, Mazarei M, Rudis MR, Fethe MH, Stewart CN: Rapid in vivo analysis of synthetic promoters for plant pathogen phytosensing. BMC Biotech 2011, 11:108.

6. Christensen $\mathrm{AH}$, Sharrock RA, Quail PH: Maize polyubiquitin genes: structure, thermal perturbation of expression and transcript splicing, and promoter activity following transfer to protoplasts by electroporation. Plant Mol Biol 1992, 18:675-689.

7. Hartmann U, Valentine WJ, Christie JM, Hays J, Jenkins GI, Weisshaar B: Identification of UV/blue light-response elements in the Arabidopsis 
thaliana chalcone synthase promoter using a homologous protoplast transient expression system. Plant Mol Biol 1998, 36:741-754.

8. Pitzschke A, Persak H: Poinsettia protoplasts - a simple, robust and efficient system for transient gene expression studies. Plant Methods 2012, 8:14

9. Hernandez-Garcia CM, Bouchard RA, Rushton PJ, Jones ML, Chen X, Timko MP, Finer JJ: High level transgenic expression of soybean (Glycine max) GmERF and Gmubi gene promoters isolated by a novel promoter analysis pipeline. BMC Plant Biol 2010, 10:237.

10. Rolfe $S A$, Tobin EM: Deletion analysis of a phytochrome-regulated monocot rbcS promoter in a transient assay system. Proc Natl Acad Sci U S A 1991, 88(2):683-2686.

11. Cazzoneli $\mathrm{Cl}$, Velten J: In vivo characterization of plant promoter element interaction using synthetic promoters. Transgenic Res 2008, 17:437-457.

12. Van Moerkercke A, Haring MA, Schuurink RC: The transcription factor EMISSION OF BENZENOIDS II activates the MYB ODORANT1 promoter at a MYB binding site specific for fragrant petunias. Plant J 2011, 67:917-928.

13. Bogs J, Jaffe FW, Takos AM, Walker AR, Robinson SP: The grapevine transcription factor VvMYBPA1 regulates proanthocyanidin synthesis during fruit development. Plant Physiol 2007, 143:1347-1361.

14. Santos-Rosa M, Poutaraud A, Merdinoglu D, Mestre P: Development of a transient expression system in grapevine via agro-infiltration. Plant Cell Rep 2008, 27:1053-1063.

15. Guan X, Zhao H, Xu Y, Wang Y: Transient expression of glyoxal oxidase from the Chinese wild grape Vitis pseudoreticulata can suppress powdery mildew in a susceptible genotype. Protoplasma 2011, 248:415-423.

16. Zottini M, Barizza E, Costa A, Formentin E, Ruberti C, Carimi F, Lo Schiavo F: Agroinfiltration of grapevine leaves for fast transient assays of gene expression and for long-term production of stable transformed cells. Plant Cell Rep 2008, 27:845-853.

17. Bertazzon N, Raiola A, Castiglioni C, Gardiman M, Angelini E, Borgo M, Ferrari S: Transient silencing of the grapevine gene VvPGIP1 by agroinfiltration with a construct for RNA interference. Plant Cell Rep 2012, 31:133-143.

18. Mizoi J, Shinozaki K, Yamaguchi-Shinozaki K: AP2/ERF family transcription factors in plant abiotic stress responses. Biochim Biophys Acta 1819, 2012:86-96

19. Wisniewski M, Nassuth A, Teulieres C, Marque C, Rowland J, Cao P-B, Brown A: Genomics of cold hardiness in woody plants. Crit Rev Plant Sci 2013, 33:92-124.

20. Stockinger EJ, Gilmour SJ, Thomashow MF: Arabidopsis thaliana CBF1 encodes an AP2 domain-containing transcriptional activator that binds to the Crepeat/DRE, a cis-acting DNA regulatory element that stimulates transcription in response to low temperature and water deficit. Proc Natl Acad Sci U S A 1997, 94:1035-1040.

21. Gilmour SJ, Zarka DG, Stockinger EJ, Salazar MP, Houghton JM, Thomashow MF: Low temperature regulation of the Arabidopsis CBF family of AP2 transcriptional activators as an early step in cold-induced COR gene expression. Plant J 1998, 16:433-442.

22. Liu Q, Kasuga M, Sakuma Y, Abe H, Miura S, Yamaguchi-Shinozaki K, Shinozaki K: Two transcription factors, DREB1 and DREB2, with an EREBP/AP2 DNA binding domain separate two cellular signal transduction pathways in drought- and low temperature- responsive gene expression, respectively, in Arabidopsis. Plant Cell 1998, 10:1391-1406.

23. Kasuga M, Liu Q, Miura S, Yamaguchi-Shinozaki K, Shinozaki K: Improving plant drought, salt, and freezing tolerance by gene transfer of a single stress-inducible transcription factor. Nature Biotech 1999, 17:287-291.

24. Miura K, Furumoto T: Cold signaling and cold response in plants. Int J Mol Sci 2013, 14:5312-5337.

25. Gao M-J, Allard G, Byass L, Flanagan AM, Singh J: Regulation and characterization of four CBF transcription factors from Brassica napus. Plant Mol Biol 2002, 49:459-471.

26. Xue G-P: Characterisation of the DNA-binding profile of barley HvCBF using an enzymatic method for rapid, quantitative and high-throughput analysis of the DNA-binding activity. Nucl Acids Res 2002, 30:e77.

27. Tong Z, Hong B, Yang Y, Li Q, Ma N, Ma C, Gao J: Overexpression of two chrysanthemum DgDREB1 group genes caused delayed flowering or dwarfism in Arabidopsis. Pl Mol Biol 2009, 71:115-129.

28. Seki M, Narusaka M, Ishida J, Nanjo T, Fujita M, Oono Y, Kamiya A, Nakajima M, Enju A, Sakurai T, Satou M, Akiyama K, Taji T, Yamaguchi-Shinozaki K, Caminci P, Kawai J, Hayashikazi Y, Shinozaki K: Monitoring the expression profiles of
7000 Arabidopsis genes under drought, cold and high-salinity stresses using a full-length cDNA microarray. Plant J 2002, 31:279-292.

29. Maruyama K, Sakuma Y, Kasuga M, Ito Y, Seki M, Goda H, Shimada Y, Yoshida S, Shinozaki K, Yamaguchi-Shinozaki K: Identification of cold-inducible downstream genes of Arabidopsis DREB1A/CBF3 transcriptional factor using two microarray systems. Plant J 2004, 38:982-993.

30. Xiao H, Siddiqua M, Braybrook S, Nassuth A: Three grape CBF/DREB1 genes are regulated by low temperature, drought and abscisic acid. Plant Cell Environ 2006, 29:1410-1421.

31. Xiao H, Tattersall E, Siddiqua M, Cramer GR, Nassuth A: CBF4 is a unique member of the CBF transcription factor family of Vitis vinifera and Vitis riparia. Plant Cell Environ 2008, 31:1-10.

32. Wang S, Yang S, Yin Y, Guo X, Wang S, Hao D: An in silico strategy identified the target gene candidates regulated by dehydration responsive element binding proteins (DREBs) in Arabidopsis genome. Plant Mol Biol 2009, 69:167-178.

33. Horstmann V, Huether CM, Jost W, Reski R, Decker EL: Quantitative promoter analysis in Physcomitrella patens: a set of plant vectors activating gene expression within three orders of magnitude. BMC Biotech 2004, 4:13.

34. Narumi O, Mor S, Boku S, Tsuji Y, Hashimoto N, Nishikawa S-I, Yokata Y: OUT, a novel basic helix-loop-helix transcription factor with an Id-like inhibitory activity. J Biol Chem 2000, 275:3510-3521.

35. Ohta S, Mita S, Hattori T, Nakamura K: Construction and expression in tobacco of a $\beta$-glucuronidase (GUS) reporter gene containing an intron within the coding sequence. Plant Cell Physiol 1990, 31:805-814.

36. Vancanneyt G, Schmidt R, O'Connor-Sanchez A, Willmitzer L, Rocha-Sosa M: Construction of an intron-containing marker gene: splicing of the intron in transgenic plants and its use in monitoring early events in Agrobacterium-mediated plant transformation. Mol Gen Genet 1990, 220:245-250.

37. Jefferson R, Kavanagh T, Bevan M: GUS fusions: $\beta$-glucuronidase as a sensitive and versatile gene fusion marker in higher plants. EMBO J 1987, 6:3901-3907.

38. Samac DA, Tesfaye M, Dornbusch M, Saruul P, Temple SJ: A comparison of constitutive promoters for expression of transgenes in alfalfa (Medicago sativa). Transgenic Res 2004, 13:349-361.

39. Chalfie MM, Tu Y, Euskirchen G, Ward WW, Prasher DC: Green fluorescent protein as a marker for gene expression. Science 1994, 263:802-805.

40. Leffel S, Mabon S, Stewart N: Applications of green fluorescent protein in plants. Biotechniques 1997, 23:912-918.

41. Ow D, Wood K, DeLuca M, DeWet J, Helsinki D, Howell S: Transient and stable expression of the firefly luciferase gene in plant cells and transgenic plants. Sci 1986, 234:856-859.

42. Mayerhofer R, Langridge W, Cormier M, Szalay A: Expression of recombinant Renilla luciferase in transgenic plants results in high levels of light emission. Plant J 1995, 7:1031-1038.

43. Mankin S, Allen G, Thompson W: Introduction of a plant intron into the luciferase gene of Photinus pyralis. Plant Mol Biol Rep 1997, 15:186-196.

44. Cazzoneli Cl, Christopher I, Velten J: Construction and testing of an intron-containing luciferase reporter gene from Renilla reniformis. Plant Mol Biol Rep 2003, 21:271-280.

45. Behre G, Smith LT, Tenen DG: Use of a promoterless Renilla luciferase vector as an internal control plasmid for transient co-transfection assays of Ras-mediated transcription activation. Biotechniques 1999, 26:24-26.

46. Odell JT, Nagy F, Chua NH: Identification of DNA sequences required for activity of the cauliflower mosaic virus 35 S promoter. Nature 1985, 313:810-812

47. Benfey $\mathrm{P}$, Chua $\mathrm{N}$ : The cauliflower mosaic virus $35 \mathrm{~S}$ promoter: combinatorial regulation of transcription in plants. Science 1990, 250:959-966.

48. Cazzonelli $\mathrm{Cl}$, Burke J, Velten J: Functional characterization of the geminiviral conserved late element (CLE) in uninfected tobacco. Plant Mol Biol 2005, 58:465-481.

49. Eini O, Yang N, Pyvovarenko T, Pillman K, Bazanova N, Tikhomirov N, Eliby S, Shirley N, Sivasankar S, Tingey S, Langridge P, Hrmova M, Lopato S: Complex regulation by apetela2 domain-containing transcription factors revealed through analysis of the stress responsive TdCor410b promoter from Durum wheat. Plos One 2013, 8:e58713.

50. Siddiqua M, Nassuth A: Vitis CBF1 and Vitis CBF4 differ in their effect on Arabidopsis abiotic stress tolerance, development and gene expression. Plant Cell Env 2011, 34:1345-1359. 
51. Sakuma Y, Liu Q, Dubouzet JG, Abe H, Shinozaki K, Yamaguchi-Shinozaki K: DNA-binding specificity of the ERF/AP2 domain of Arabidopsis DREBs, transcription factors involved in dehydration- and cold-inducible gene expression. Biochem Biophys Res Comm 2002, 290:998-1009.

52. Hao D, Ohme-Takagi M, Sarai A: Unique mode of GCC box recognition by the DNA-binding domain of ethylene-responsive element-binding factor (ERF domain) in plant. J Biol Chem 1998, 273:26857-26861.

53. Tuller T, Waldman YY, Kupiec M, Ruppin E: Translation efficiency is determined by both codon bias and folding energy. Proc Natl Acad Sci U S A 2010, 107:3645-3650.

54. Höfgen R, Willmitzer L: Storage of competent cells for Agrobacterium transformation. Nucleic Acids Res 1988, 16:9877.

55. Bendahmane A, Kanyuka K, Baulcombe DC: The Rx gene from potato controls separate virus resistance and cell death responses. Plant Cell 1999, 11:781-791.

56. Vaucheret $\mathrm{H}$ : Promoter-dependent trans-inactivation in transgenic tobacco plants; kinetic aspects of gene silencing and gene reactivation. C R Acad Sci 1994, 317:310-323.

57. Bradford M: A rapid and sensitive method for the quantitation of microgram quantities of protein utilizing the principle of protein-dye binding. Anal Biochem 1976, 72:248-254.

doi:10.1186/1746-4811-10-32

Cite this article as: Nassuth et al: Newly developed quantitative transactivation system shows difference in activation by Vitis CBF transcription factors on DRE/CRT elements. Plant Methods 2014 10:32.

\section{Submit your next manuscript to BioMed Central and take full advantage of:}

- Convenient online submission

- Thorough peer review

- No space constraints or color figure charges

- Immediate publication on acceptance

- Inclusion in PubMed, CAS, Scopus and Google Scholar

- Research which is freely available for redistribution 